\title{
Evolution of reproductive systems in filamentous ascomycetes. II. Evolution of hermaphroditism and other reproductive strategies
}

\author{
M. J. NAUTA \& R. F. HOEKSTRA \\ Department of Genetics, Agricultural University, Dreyenlaan 2, 6703 HA Wageningen, The Netherlands
}

\begin{abstract}
The evolution of different reproductive systems in filamentous ascomycetes is studied in a population genetic model. These fungi differ essentially from higher plants and animals because mating types can exist in addition to male and female gametes, and the conidia serve as both male gametes and asexual spores; moreover, selfing is genetically equivalent to asexual reproduction in these haploid organisms. A variable fitness of ascospore production is predicted as the explanation for the evolution of two systems that abundantly exist in nature: hermaphroditism in heterothallic species and the formation of both asexual and sexual spores in homothallic species. Imperfect fungi will evolve if sexual spores do not show a remarkably higher fitness than asexual spores.
\end{abstract}

Keywords: ascomycetes, dioecy, evolution, hermaphroditism, reproduction.

\section{Introduction}

A great variety of reproductive systems exist in filamentous ascomycetes. Compared with the reproductive systems in higher plants, some specific differences attract attention. The first is the possible occurrence of mating types in addition to the existence of both male and female gametes [discussed in a previous study Nauta \& Hoekstra, 1992], where only crossings between male and female with unlike mating types are possible. The second is that both sexual and asexual spores exist, while the asexual spores (conidia) can often also function as male gametes. Finally the fact that the gamete-producing individual is haploid has special implications for selfing and inbreeding.

Mating systems such as monoecy, dioecy, gynodioecy and trioecy are known in plants. The evolutionary forces that can account for these different types have been thoroughly studied (Charlesworth \& Charlesworth, 1978a, b; Charlesworth \& Ganders, 1979; Gregorius et al., 1982, 1983; Ross, 1982): for fungi, however, such studies appear to be lacking.

This paper is a theoretical analysis of the evolutionary relations between a number of fungal reproductive strategies. Attention is mainly limited to the family of Sordariaceae, which contains a number of well-studied species such as Neurospora crassa and Sordaria fimicola; many ascomycete mating systems are represented.

All heterothallic Sordariaceae investigated to date have both conidia (male gametes) and ascogonia (structures holding the female gamete) and can therefore be considered to be hermaphrodite. No report on a natural dioecious filamentous ascomycete (i.e. with seperate male and female individuals) is known to us. This leads to the question why, in Sordariaceae, hermaphrodites with mating types do occur, whereas separate males and females without mating types, do not.

It is also remarkable that in many homothallic species (such as the homothallic Neurosporas and Sordaria fimicola) the conidia are absent and ascogonia no longer form trichogynes (small hyphae growing towards fertilizing conidia). This means that no asexual spores are formed and outcrossing is probably very rare, being only possible after heterokaryosis.

Another phenomenon in fungi, incomparable with higher plants, is the existence of imperfect fungi, in which no sexual stage is found. Although taxonomically they are not classified as ascomycetes, most can 


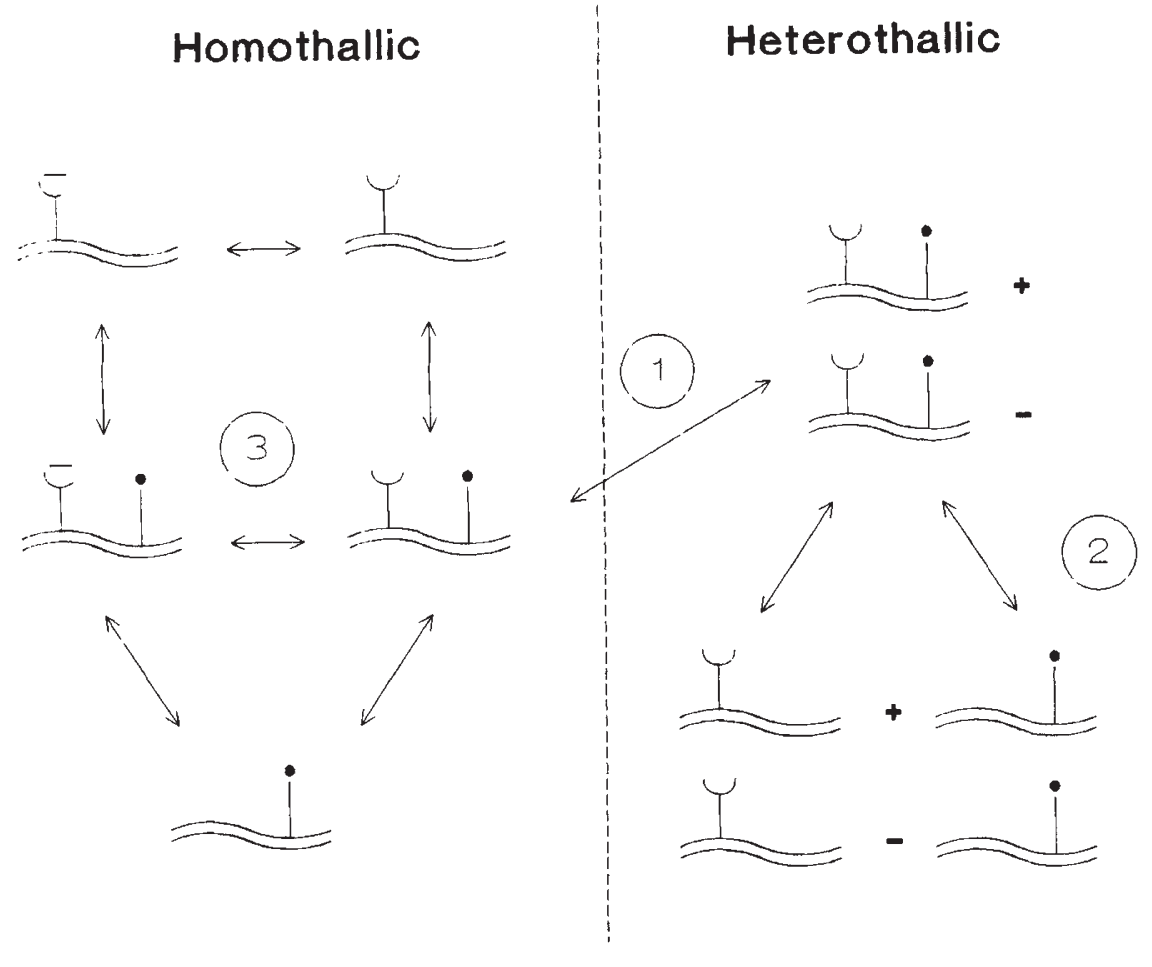

Fig. 1 Overview of the evolutionary transitions considered. Conidia are symbolized by a dot above a stalk, ascogonia by a cup on a stalk (ready to capture airborne conidia). Strictly selfing ascogonia that are not fertilized by conidia are symbolized by a cup covered with a lid. Arrows indicate possible transitions between 1 homothallics and heterothallics (Nauta \& Hoekstra, 1992), (2) hermaphrodite, male and female heterothallics (Section A), (3) different types of homothallics (Section B). be considered as such because they show all the characteristics of ascomycetes except a sexual cycle (e.g. Ainsworth, 1973). These imperfect fungi can be considered as pure male ascomycetes, which only form conidia and no ascogonia. As there are no more females to fertilize, the conidia have ceased to function as male gametes and are now specialized asexual spores.

A model is developed in this study in an attempt to find evolutionary pathways for all these different mating systems (as illustrated in Fig. 1). The important fitness parameters are the differences between sexual and asexual reproduction and between selfing and outbreeding.

\section{The model}

The model organism in this study is the idealized ascomycete described previously (Nauta \& Hoekstra, 1992). It has a haploid life cycle and in principle can form both conidia and ascogonia. In the young ascus, karyogamy and meiosis take place and the ascospores are formed. The population size is assumed to be infinite and generations are separated.

It is assumed that the conidia and ascospores formed are dispersed randomly over the habitat of the population. They can land on three different types of substrate: (1) on an appropriate site where they can germinate, (2) on an unfit site where they cannot survive, or (3) on a site which is already occupied by another individual. In the last case a landing conidium can fertilize an ascogonium of that individual, whereas a landing ascospore will get lost. It is assumed that there are always sufficient conidia in the population to fertilize all available ascogonia.

The 'twofold disadvantage of sex' (Maynard Smith, 1971) or 'cost of genome dilution' (Lewis, 1987) is manifested in the production of ascospores. In the ascospores half of the genes are of paternal origin (the conidium) and half of maternal origin (the ascogonium), while in an asexual spore all genes are from 'paternal' origin. Although half of its genome is lost, a conidium may profit by sexual reproduction, because the female parent provides the resources to produce several (normally eight) ascospores out of one conidium. The female parent, however, experiences a twofold cost of sex, compared to a situation where she would produce asexual spores or selfed ascospores. It is essential, therefore, that ascospores, being more resistant and capable of remaining viable for long periods, have a higher fitness than asexual spores (Perkins \& Turner, 1988). In addition, some kind of inbreeding depression must occur to explain outbreeding.

Several types of individual are considered. They differ in the production of male and female gametes and in the frequency of self-fertilization. An individual that produces only conidia (a 'male') forms $N$ conidia, 
and an individual that produces only ascogonia (a 'female') forms $n$ ascogonia. A hermaphrodite forms $\alpha N$ conidia and $\beta n$ ascogonia. As the energy required to form a conidium (just a small cell) must be much lower than that needed to form an ascogonium (the receptive structure) plus a fruiting body filled with ascospores, we assume $n \ll N$. If all individuals can use the same amount of energy for reproduction, then $\alpha+\beta=1$ for all hermaphrodites. This is assumed in the model, so we may define $\alpha=z$ and $\beta=1-z$, where $z$ is the 'maleness' of the individual.

The selfing rate for homothallic species is $s$. Outbred and selfed progeny have a relative fitness of 1 and $d$ respectively.

As elaborated in Appendix I, a general recursion equation can be derived from these assumptions. It is shown that the differences in fitness between sexual and asexual spores can be summarized by a single parameter $\theta$ for the fitness of ascospore production.

When a population contains different types of individuals, where type $j$ has a frequency $x_{j}$, a maleness $z_{j}$ and a selfing rate $s_{j}$, this general recurrence relation is given by:

$$
\begin{aligned}
W x_{j}^{\prime}= & x_{j}\left\{z_{j}\left[1+\frac{1}{2} \theta \frac{\sum_{k}\left(1-s_{k}\right)\left(1-z_{k}\right) x_{k}}{\sum_{k} z_{k} x_{k}}\right]\right. \\
& \left.+\frac{1}{2} \theta\left(1-z_{j}\right)\left[1-s_{j}(1-2 d)\right]\right\}
\end{aligned}
$$

where

$$
W=(1-\theta) \sum_{k} x_{k} z_{k}+\theta\left[1-(1-d) \sum_{k}\left(1-z_{k}\right) s_{k} x_{k}\right] \text {. }
$$

Note that the model covers both homo- and heterothallic populations, but not populations polymorphic for this trait.

In subsequent sections a number of specific models are analysed. The different types of individual and the possible transitions between them are shown schematically in Fig. 1. A constant and a variable ascospore fitness parameter $\theta$ are considered.

\section{A1 Heterothallic population with males, females and hermaphrodites. Constant ascopore fitness $\theta$}

Consider a population with three types $j, j=1$ representing males $\left(z_{1}=1, s_{1}=0\right), j=2$ representing hermaphrodites $\left(z_{2}=z, s_{2}=0\right)$ and $j=3$ representing females $\left(z_{3}=0, s_{3}=0\right)$. From the resulting recurrence relations

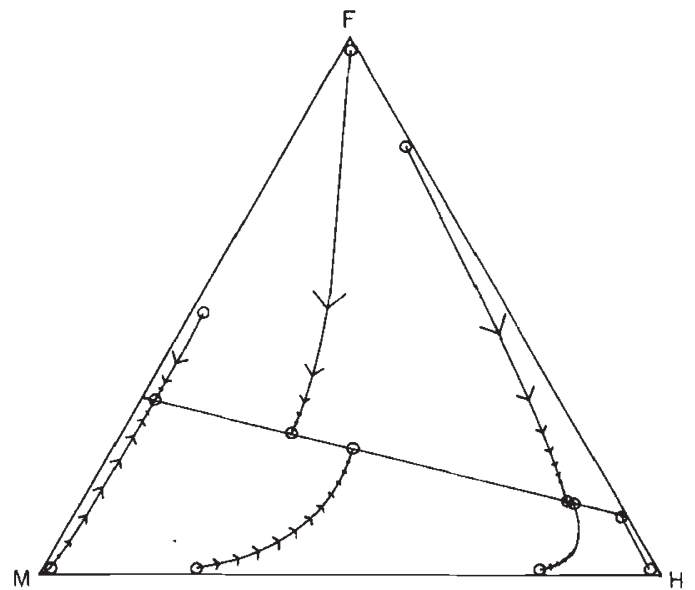

Fig. 2 Results for heterothallism with constant ascospore fitness $\theta$, as described in section $\mathrm{Al}$, with $\theta=4$ and $z=0.75$. Frequencies of males $(\mathrm{M})$, females $(\mathrm{F})$, and hermaphrodites $(\mathrm{H})$ are given in a de Finetti diagram. Starting with different frequencies $x_{j}$, stability is reached when $\left.x_{1}+z x_{2}=\frac{1}{2} \theta / \theta-1\right)$, given as a solid line in the diagram. Arrows indicate the frequency every subsequent generation.

(see Appendix II) it can be deduced that the population is in equilibrium if

$x_{1}+z x_{2}=\frac{\frac{1}{2} \theta}{\theta-1}$.

It is easy to see that for $\theta \leq 2$ a pure male population is stable. This implies that with $\theta \leq 2$ the evolution of imperfect fungi is expected. For $\theta>2$ polymorphism is stable; the equilibrium values of $x_{j}$ depend on the starting frequencies and are located on a straight line segment (Fig. 2). Thus, in an infinite population, one should expect to find trioecy: populations with hermaphrodites, males and females.

In a finite population random genetic drift may cause the frequencies in the population to change along the equilibrium line (2). As stated in Appendix II no specific tendency towards dioecy of hermaphroditism could be found, so in the long-term a finite population may either become dioecious (males and females) or consist of hermaphrodites with either males or females.

\section{A2 Heterothallic population with males, females and hermaphrodites. Variable ascospore fitness $\theta$}

Sexual spores are often formed under conditions of stress alone. In the model this can be represented by a varying $\theta$. It is assumed that the fitness of ascospores is rather low for many generations but occasionally very high. This situation has been studied numerically. An 


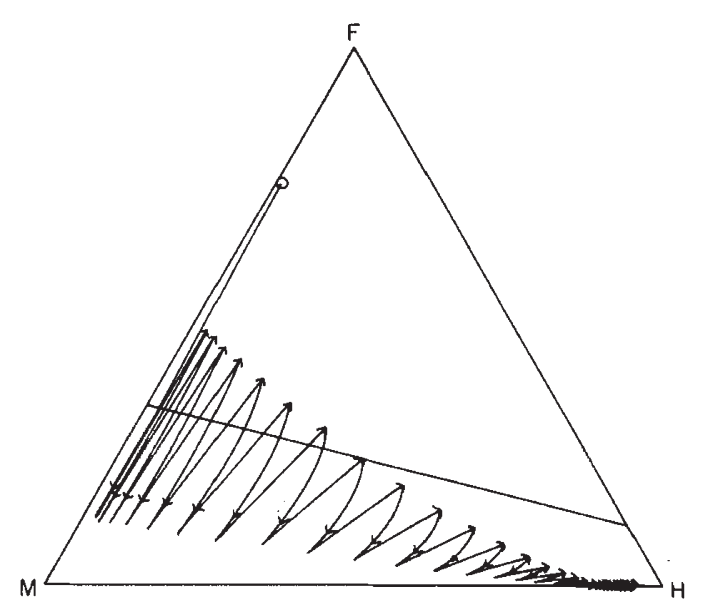

Fig. 3 Results for heterothallism with variable ascospore fitness $\theta$, as described in section $A 2$, with $\theta_{G}=4$ and $z=0.75$. Every tenth generation $\theta=1000$. The line $x_{1}+z x_{2}=\frac{1}{2} \theta_{\mathrm{G}} /$ $\left(\theta_{\mathrm{G}}-1\right)$ is given as in Fig. 2. Arrows indicate frequencies every fifth generation. Finally a polymorphism of hermaphroditism and males is reached.

example of the results, where $\theta=2.166$ for nine generations and $\theta=1000$ every tenth generation (so the geometric mean $\theta_{\mathrm{G}}=4$ ), is illustrated in Fig. 3. Due to the fluctuations the hermaphrodite frequency slowly increases.

It has been found that, unlike a constant $\theta$, a varying $\theta$ causes trioecy to be unstable. If $\theta_{i}$ represents the value of $\theta$ in generation $i$, the resulting population will be pure male, i.e. imperfect if

$\prod_{i}\left[z+\frac{1}{2} \theta_{i}(1-z)\right]<1$

If this is not true the population will become polymorphic hermaphrodite/male or hermaphrodite/ female, or will become purely hermaphroditic. The conditions for these different results are given in Appendix II.

The fate of different hermaphrodites (with different values of $z_{j}$ ), occurring simultaneously in the population, has been investigated in some additional numerical studies. Our calculations show that such a situation is rather complex. The equilibrium frequencies for the different types of hermaphrodite partly depend on starting frequencies, and a number of hermaphrodite types can invade under a variety of circumstances. If hermaphrodites with any possible value of $z_{j}$ can exist, however, the population will always become purely hermaphroditic, polymorphic for $z_{i}$.

\section{B1 Homothallic population with selfing, non- selfing, conidiating and non-conidiating types. Constant ascospore fitness $\theta$}

Self-fertilization is possible in a homothallic population, so $s_{j}$ will have values greater than 0 . As emphasized before, most homothallic species do not form trichogynes, so the conidia (if present) will hardly be able to fertilize the ascogonia. It is assumed that there can be two types of pure selfer $\left(s_{5}=s_{5}=1\right)$, both with $\left(z_{4}=z\right)$ and without $\left(z_{5}=0\right)$ conidia. Fertilization occurs internally in these types by fusion of two (usually genetically identical) nuclei within an ascogenous hypha, as for example in Sordaria fimicola and Aspergillus nidulans.

In addition to these two types of individuals, males, females and hermaphrodites are also considered. The latter now have the ability to self fertilize $\left(s_{2}=s\right)$.

The five types: $j=1-5$ are symbolized by $\mathbf{i}, \mathbf{u i}, \mathbf{u}$, $\hat{\mathbf{u}} \mathbf{i}$ and $\mathbf{u}$ in accordance with the symbols used in Fig. 1; $\mathbf{i}$ stands for a conidiophore with a conidium and $\mathbf{u}$ stands for the receptive structure of the ascogonium, which is blocked in a strictly selfing $\hat{\mathbf{u}}$. An overview is given in Table 1 and the recurrence relations are shown in Appendix III.

Analysis of the recurrence relations shows that the values of the parameters $\theta$ and $d$ are important in determining which types of individual can form a stable population.

If the fitness, $d$, of selfed offspring is smaller than

Table 1 A survey of the types used in the model. (The genus Aspergillus does not belong to the Sordariaceae, but the species mentioned seem to fit the model well.)

\begin{tabular}{llllll}
\hline$j$ & Name & & & & Well known \\
example species
\end{tabular}


0.5 , the strictly selfing types, $\hat{\mathbf{u}}$ and $\mathbf{u} \mathbf{i}$, will disappear; the situation is then comparable to the cases described in section A1. All hermaphrodites with $s_{2}>0$ will disappear, however, and a stable population will contain only males (if $\theta \leq 2$ ) or only males and females (with possibly hermaphrodites with $s_{2}=0$ ) (if $\theta>2$ ).

It is easy to see, from a consideration of the recurrence relations in Appendix III, that for $d>0.5$ the strictly selfing types $\hat{\mathbf{u}}$ and $\mathbf{u} i$ always have a higher fitness than those fertilized by conidia, $\mathbf{u}$ and ui. Competition between $\hat{\mathbf{u}}$, $\hat{\mathbf{u}} \mathbf{i}$ and $\mathbf{i}$ will result in a population either monomorphic for $\hat{\mathbf{u}}$ (if $\theta d>1$ ) or monomorphic for $\mathbf{i}$ (see Appendix III). Thus the model suggests that a homothallic species like Aspergillus nidulans, which forms conidia that serve as asexual spores, can never be stable if the ascospore fitness is constant.

\section{B2 Homothallic population with selfing, non- selfing, conidiating and non-conidiating types. Variable ascospore fitness $\theta$}

As in the heterothallic population considered in section $\mathrm{A}$, the model with a variable $\theta$ gives results that differ from those of the constant $\theta$ model.

As in section B1 where $d<0.5$, the selfing types $\hat{\mathbf{u}}$ and ûi are never stable in this population. However, some types of partly selfing hermaphrodites (ui) can now invade the population (The conditions for this are given in Appendix III). As hermaphrodites with $s_{2}=0$ are again the optimal hermaphrodite type, the situation for $d<0.5$ is apparently identical to the one described in section A2 for heterothallic populations.

The competition between $\hat{\mathbf{u}}$, ûi and $\mathbf{i}$ can be won for $d>0.5$ by each of the three types this time. As calculated in Appendix III, it can be deduced that with a variable $\theta$ (with $\theta=\theta_{\mathrm{i}}$ in generation $i$ ), a population will become monomorphic for ûi if

$\prod_{i}\left[z+\theta_{i} d(1-z)\right]>\prod_{i} \theta_{i} d$

and

$\prod\left[z+\theta_{i} d(1-z)\right]>1$.

If these inequalities are not satisfied, the population will become monomorphic for $i$ if

$\prod \theta_{i} d<1$

and monomorphic for $\hat{\mathbf{u}}$ if

$\prod_{i} \theta_{i} d>1$

A summary of the results is given schematically in Table 2 .
Table 2 Summary of the results described in sections A and B. (The notation is explained in Table 1.)

\begin{tabular}{ll}
\hline $\begin{array}{l}\text { Heterothallic } \\
\text { (Section A) }\end{array}$ & $\begin{array}{l}\text { Homothallic } \\
\text { (Section B) }\end{array}$ \\
\hline $\begin{array}{c}\text { Constant } \theta \\
\theta<2: \mathbf{i}\end{array}$ & $d<0.5:$ \\
& $\theta<2: \mathbf{i}$ \\
& $\theta>2, s \neq 0: \mathbf{i}+\mathbf{u}$ \\
$\theta>2: \mathbf{i}+\mathbf{u i}+\mathbf{u}$ & $\theta>2, s=0: \mathbf{i}+\mathbf{u i}+\mathbf{u}$ \\
& $d>0.5:$ \\
& $\theta d<1: \mathbf{i}$ \\
& $\theta d>1: \hat{\mathbf{u}}$ \\
Variable $\theta$ & \\
$\mathbf{i}[$ eq. $(3)]$ & $d<0.5:$ as Heteroth. \\
or & but disadvantage \\
$\mathbf{u i}(+\mathbf{i})(+\mathbf{u})$ & $\mathbf{u i}$ if $s \neq 0[$ eq. (19)] \\
& $d>0.5: \mathbf{i}$, \\
& ûi or \\
& $\hat{\mathbf{u}}[$ eq. $(4)]$ \\
\hline
\end{tabular}

\section{Discussion}

The model suggests that in heterothallic populations hermaphroditism can only be stable if the fitness of ascospore formation, expressed in the parameter $\theta$, is variable. With a constant $\theta$ no advantage was found for hermaphroditism above dioecy or trioecy. However, as is apparent from the precise definition of $\theta$ as given in Appendix I, a constant $\theta$ is biologically quite improbable. Variation in $\theta$ will result not only from environmental heterogeneity affecting ascospore fitness but also from variation in the fractions of germinating and fertilizing conidia. Therefore, a variable $\theta$ is the most relevant case and the evolution of hermaphroditism is expected.

The model shows for homothallism that only for a varying ascospore fitness are there conditions for stability of hermaphroditism (ui) or selfing with conidia formation ( $\hat{u} i$ ). No report has been found of the first in the homothallic Sordariaceae, and the second seems to be rare (Perkins \& Turner, 1988). This is in accordance with the model, where these outcomes are only found for rather restricted parameter combinations.

The occurrence of 'conidiating selfers', like Aspergillus nidulans, is an interesting phenomenon because both the sexual and the asexual spores are genetically identical to the parent. This coexistence of two types of spore is comparable to the seed heteromorphism found in higher plants, which can also only be explained by some environmental variation in space 
or time (Venable \& Brown, 1988). Whether meiosis, preceding ascospore formation, still has a function in these species, or should be considered a phylogenetic artifact remains to be questioned.

In a previous study (Nauta \& Hoekstra, 1992) the evolution of mating types and the competition between homo- and heterothallic types has been studied. It was found that if all outcrossing sex has equal fitness, homothallism is stable if the fitness of selfing $d>0.5$ and polymorphism for the two types is stable if $d<0.5$. Heterothallism is only stable if homothallism has some additional disadvantage.

In the current model (B1 and B2) the condition $d<0.5$ reappears. If this condition is satisfied, a nonselfing hermaphrodite is the optimal type if the ascospore fitness $\theta$ varies. It could be that heterothallism is the only way for a species to prevent selfing. A problem is that with heterothallism the frequency of potential mates is lowered. A polymorphic homo- and heterothallic population is expected (Nauta \& Hoekstra, 1992), which is unknown to exist in nature.

The 'cost of genome dilution' (Lewis, 1987), is revealed twice in the model. Both selfing and asexual reproduction are forms of uniparental inheritance in haploids, where the progeny is genetically identical to the parent. The advantage of these modes of reproduction over sexual reproduction are twofold in principle (Maynard Smith, 1971). This factor two shows up for the parameters $d$ (for selfing) and $\theta$ (for asexual reproduction), $d=0.5$ and $\theta=2$ being the threshold values in the model.

Charnov et al. (1976) and Maynard Smith (1978) studied a model for resource allocation in hermaphrodites, where males produce $N$ sperm, females produce $n$ eggs and hermaphrodites $\alpha N$ sperm and $\beta n$ eggs. They found that hermaphroditism is stable if $\alpha+\beta>1$ and dioecy if $\alpha+\beta<1$. The case of $\alpha+\beta=1$, as assumed in the current model, is a neutral case in their model. (Their findings are comparable to those found in section $\mathrm{A} 1$, where all types are neutral if the population has reached the equilibrium $\left.x_{1}+z x_{2}=\frac{1}{2} \theta /(\theta-1)\right)$. The main difference from our model is that they do not consider the dynamics of the relative frequencies of the different types in an infinite random mating population but discuss why the fitness sets for the allocation of resources to male and female functions will normally not be linear $(\alpha+\beta=1$, as in our model). One of the main reasons for assuming a convex fitness set (which makes hermaphroditism the optimal strategy) is the diminishing returns for male and female function. However, as random mating is assumed and frequencydependent aspects of the returns for males and females are covered implicitly in the formulas of our model, we could find no reason to assume such a fitness set here.
Charlesworth \& Charlesworth (1978a, b) have modelled the evolution from monoecy to dioecy via gynodioecy in angiosperms. The general formula used in their studies is:

$$
\begin{aligned}
W x_{j}^{\prime}= & x_{j}\left[\frac{1}{2} b_{j} \frac{\sum_{k}\left(1-s_{k}\right) e_{k} x_{k}}{\sum_{k} b_{k} x_{k}}\right. \\
& \left.+\frac{1}{2} e_{j}\left(1-s_{j}\right)+e_{j} s_{j}(1-\delta)\right]
\end{aligned}
$$

where

$W=\sum_{k}\left(1-s_{k}\right) e_{k} x_{k}+\sum_{k}(1-\delta) s_{k} e_{k} x_{k}$.

Here $e_{j}$ is the ovule production of type $j, b_{j}$ is the pollen production, $s_{j}$ is the fraction selfing and $1-\delta$ is the fitness of offspring from selfing.

Equation (7) is very similar to the general recursion (1) in this study. In fact, by excluding asexual reproduction by means of male gametes, and by putting:

$e_{j}=\left(1-z_{j}\right)$

$b_{j}=z_{j} \quad$ and

$1-\delta=d$,

both formulae become identical.

The simplest situation Charlesworth and Charlesworth discuss is $b_{j}=0$ and $e_{j}=1+k$ for male-steriles and $b_{j}=1$ and $e_{j}=1$ for hermaphrodites. They found that a male-sterile mutant can invade a hermaphrodite population if $k>1-2 \delta s$. This can be compared with our model if $1+k=1 /(1-z)$ and $\theta=\infty$, using types $\mathbf{u}$ (male-sterile) and ui (hermaphrodite) only. As the condition $\theta=\infty$ (meaning a fitness 0 for asexual reproduction) is unrealistic, this situation is of little biological relevance in Sordariceae.

An important difference between angiosperms and the ascomycetes considered here is that pollen differ from conidia in their inability to transport cytoplasmic genes and to germinate asexually. In addition, in plants sex determination occurs at the diploid stage. These differences make a comparison with models concerned with cytoplasmic inheritance (Charlesworth \& Ganders, 1979) and different types of biallelic sex determination (Gregorious et al., 1982, 1983) less useful.

The main selective force considered by Charlesworth \& Charlesworth (1978a, b) is inbreeding depression, which shows up after selfing. They found this necessary for gynodioecy to evolve, just as it is necessary for the evolution of heterothallism (Nauta \& 
Hoekstra, 1992). One problem is that because of haploidy in fungi, the disadvantage of selfing is hardly comparable with that in higher plants and animals. Probably this disadvantage is much lower in haploids. The haploidy and the existence of mating types in addition to hermaphroditism make it difficult to compare the evolution of hermaphroditism in angiosperms and ascomycetes.

The ability of conidia to germinate as asexual spores makes the evolution of imperfect fungi possible. It has been found that the fitness $\theta$ of ascospore production must be more than twice the fitness of asexual spores in order for imperfect fungi not to evolve. Whether (and if so, why) this presumed higher fitness of ascospores is related to sexual reproduction is unclear.

Finally we conclude that if a species is heterothallic the evolution of hermaphroditism can be explained. But for a homothallic species, even if it is suffering from inbreeding depression, both the evolution to heterothallism (Nauta \& Hoekstra, 1992) and to dioecy (defined as the existence of males next to females), are hard to explain. There therefore seems no reason why heterothallism should be more common than dioecy.

It is a problem that the many experimental data, needed for a better understanding of the evolutionary aspects of reproductive systems in ascomycetes, are lacking. More knowledge about the natural populations of ascomycetes is necessary to obtain reliable estimates of the values of the parameters related to the degree of 'maleness' of hermaphrodites $(z)$, rates of selfing $(s)$, and to fitness differences between the various types of spores $(d$ and $\theta)$. More species should be investigated to obtain more information on the reproductive processes, the factors that initiate conidia and ascogonium production, the frequency of outcrossing in homothallic and heterothallic species and the occurrence of inbreeding depression. It would be very interesting to carry out a thorough search for polymorphisms in homo-and heterothallism or in sex types. The current models predict that such polymorphisms may be found in nature.

\section{Acknowledgements}

The authors wish to thank an anonymous referee for useful comments. These investigations were supported by the Foundation for Biological Research (BION), which is subsidized by the Netherlands Organization for Scientific Research (NWO; grant no. 811-439-085).

\section{References}

AINSWORTH, G. C. 1973. Introduction and keys to higher taxa. In: Ainsworth, G. C., Sparrow, F. K. and Sussman, A. S. (eds), The Fungi. An Advanced Treatise, Volume IVa, Academic Press, New York, pp. 1-7.

CHARLESWORTH, B. AND CHARLESWORTH, D. 1978a. A model for the evolution of dioecy and gynodioecy. Am. Nat., 112, 975-997.

ChaRlesworth, D. AND CHARLESWORTH, B. 1978b. Population genetics of partial male-sterility and the evolution of monoecy and dioecy. Heredity, 41, 137-153.

CHARLESWORTH, D. AND GANDERS, F. R. 1979. The population genetics of gynodioecy with cytoplasmic-genic malesterility. Heredity, 43, 213-218.

CHARNOV, E. L., MAYNARD SMITH, J. AND BULL, J. J. 1976. Why be an hermaphrodite? Nature, 263, 125-126.

GREGORIUS, H.-R., ROSS, M. D. AND GILLET, E. M. 1982. Selection in plant populations of effectively infinite size. III. The maintenance of females among hermaphrodites for a biallelic model. Heredity, 48, 329-343.

GREGORIUS, H.-R., ROSS, M. D. AND GILLET, E. M. 1983. Selection in plant populations of effectively infinite size. V. Biallelic models of trioecy. Genetics, 103, 529-544.

LEWIS, W. M., Jr. 1987. The cost of sex. In: Stearns, S. C. (ed.), The Evolution of Sex and its Consequences, Birkhäuser Verlag, Basel, pp. 33-57.

MAYNARD SMITH, J. 1971. The origin and maintenance of sex. In: Williams, G. C. (ed.), Group Selection, Chicago, Aldine-Atherton, pp. 163-175.

MAYNARD SMITH, J. 1978. The Evolution of Sex, Cambridge University Press, Cambridge.

NAUTA, M. J. AND HOEKSTRA, R. F. 1992. Evolution of reproductive systems in filamentous ascomycetes. I. Evolution of mating types. Heredity, 68, 405-410.

PERKINS, D. D. AND TURNER, B. C. 1988 . Neurospora from natural populations: Towards the population biology of a haploid eukaryote. Exp. Mycol., 12,91-131.

ROss, M. D. 1982. Evolutionary pathways to subdioecy. Am. Nat., 119, 297-318.

VENABLE, D. L. AND BROWN, J. S. 1988. The selective interactions of dispersal, dormancy and seed size as adaptations for reducing risk in variable environments. Am. Nat., 131, 360-384.

\section{Appendix}

\section{Derivation of the general formula (1)}

Consider an individual model organism of type $j$, which produces $\alpha_{j} N$ conidia and $\beta_{j} n$ ascogonia. Then, if each ascogonium produces $c$ ascospores, an individual of type $j$ produces $\beta_{i} c n$ ascospores. (As ascospore poduction requires higher energy expenditure than conidium production, $c n<N$.)

As described in the main text, the different spores can land on different substrates. A fraction $p_{\text {a }}$ germinates from the produced ascospores and a fraction $1-p_{\mathrm{a}}$ is lost. A fraction $1-p_{\mathrm{c}}$ is lost from the conidia, a fraction $p_{\mathrm{c}} \cdot E$ germinates as asexual spore and a fraction $p_{c} \cdot(1-E)$ serves as a male gamete. (As 
ascospores normally have a greater survival ability $p_{\mathrm{a}}>p_{\mathrm{c}} \cdot E$.)

It is assumed that a fraction $s_{j}$ of the ascogonia are self-fertilized and that the remaining ascogonia are (randomly) cross-fertilized. The amount of conidia used for selfing is assumed to be negligible.

There is a total of $M$ conidia and $m$ ascogonia in the whole population that participate in outcrossing. When the relative fitness of an asexual spore is scaled equal to 1 , the fitness of a sexual spore is assumed to be equal to $F$ if it results from outcrossing, and $F d$ if it results from selfing.

Thus the genetic contribution by this individual to the next generation is

$$
\begin{aligned}
& \left(\alpha_{j} N p_{\mathrm{c}} E\right)+\left[\frac{1}{2} F p_{\mathrm{a}} \beta_{j} c n\left(1-s_{j}\right)\right]+\left(F p_{\mathrm{a}} d \beta_{j} c n s_{j}\right) \\
& \quad+\left[\frac{1}{2} F p_{\mathrm{a}} p_{\mathrm{c}}(1-E) \alpha_{i} N c m / M\right],
\end{aligned}
$$

where $\alpha_{j} N p_{\mathrm{c}} E$ is the number of successful asexual spores, $\frac{1}{2} F p_{\mathrm{a}} \beta_{j} \operatorname{cn}\left(1-s_{j}\right)$ is the number of successful ascospores from cross-fertilization, $F p_{\mathrm{a}} d \beta_{j} c n s_{j}$ is the number of successful ascospores from self-fertilization, and ${ }_{2}^{1} F p_{\mathrm{a}} p_{\mathrm{c}}(1-E) \alpha_{j} N c m / M$ is the number of successful ascospores (from other individuals) resulting from fertilization by conidia from the individual considered.

Thus the change in frequency $x_{j}\left(\Sigma x_{j}=1\right)$ of type $j$ will be given by

$$
\begin{aligned}
V x_{j}^{\prime}==_{j} & \cdot\left[\alpha_{j} N\left(p_{\mathrm{c}} E+\frac{1}{2} F p_{\mathrm{a}} p_{\mathrm{c}}(1-E) c_{\mathrm{M}}\right)\right. \\
& \left.+\frac{1}{2} p_{\mathrm{a}} \beta_{j} c n\left(2 s_{j}+1-s_{j}\right)\right]
\end{aligned}
$$

where $V$ equals the sum of the right-hand sides of all $j$.

By putting

$\theta=\frac{F p_{\mathrm{a}} c n}{N p_{\mathrm{c}} E}$

and noting that

$$
\frac{m}{M}=\frac{n \cdot \sum_{j}\left(1-s_{j}\right) \beta_{j} x_{j}}{N p_{c}(1-E) \cdot \sum_{j} \alpha_{j} x_{j}}
$$

equation $(8)$ can be rewritten as

$$
\begin{aligned}
W x_{j}^{\prime}= & x_{j} \cdot\left[\alpha_{j}\left(1+\frac{1}{2} \theta \frac{\sum_{k}\left(1-s_{k}\right) \beta_{k} x_{k}}{\sum_{k} \alpha_{k} x_{k}}\right)\right. \\
& \left.+\frac{1}{2} \theta \beta_{j}\left(2 s_{j} d+1-s_{j}\right)\right]
\end{aligned}
$$

where $W$ equals the sum of the right-hand sides. (1).

For $\alpha_{j}=z_{j}$ and $\beta_{j}=\left(1-z_{j}\right)$ formula $(10)$ is equal to
In the analysis the parameter $\theta$ appears to be very important. Thus from equation (9) it follows that

$\theta=\frac{F_{\mathrm{ra}} \times P_{\mathrm{ea}}}{P_{\mathrm{ec}}}$

where $F_{\mathrm{ra}}$ is the relative ascospore fitness, $P_{\mathrm{ea}}$ and $P_{\mathrm{ec}}$ are the effective ascospore and condidium production, respectively. So $\theta$ can be called the fitness of ascospore production.

\section{The dynamics of the system of recurrence rela- tions in a heterothallic population}

For the three types $j$ considered in section $\mathrm{A} 1$ of this study $(j=1,2$ and 3 representing respectively males, hermaphrodites and females) formula (1) becomes:

$$
\begin{aligned}
& W x_{1}^{\prime}=x_{1}\left(1+\frac{1}{2} \theta \frac{(1-z) x_{2}+x_{3}}{x_{1}+z x_{2}}\right) \\
& W x_{2}^{\prime}=x_{2}\left\{z\left[1+\frac{1}{2} \theta \frac{(1-z) x_{2}+x_{3}}{x_{1}+z x_{2}}\right]+\frac{1}{2} \theta(1-z)\right\}
\end{aligned}
$$

$W x_{3}^{\prime}=x_{3} \frac{1}{2} \theta$

where,

$$
W=(1-\theta)\left(x_{1}+z x_{2}\right)+\theta \text {. }
$$

Defining $x_{1}+z x_{2}=y$ and $\frac{1}{2} \theta /(\theta-1)=z^{*}$, these equations can be rewritten as:

$$
\begin{aligned}
& \Delta x_{1}=x_{1} \frac{(y-1)\left(y-z^{*}\right)}{y\left(2 z^{*}-y\right)} \\
& \Delta x_{2}=x_{2} \frac{(y-z)\left(y-z^{*}\right)}{y\left(2 z^{*}-y\right)} \\
& \Delta x_{3}=x_{3} \frac{y-z^{*}}{2 z^{*}-y}
\end{aligned}
$$

It is now easy to see that the population is in equilibrium if $y=z^{*}$, so if $x_{1}+z x_{2}=\frac{1}{2} \theta /(\theta-1), z^{*}$ can be interpreted as the 'maleness' of the whole population in equilibrium. Furthermore, it is easy to see that

$x_{1}$ decreases if $y>z^{*}$ and else increases, $x_{3}$ decreases if $y<z^{*}$ and else increases and $x_{2}$ decreases if $z<y<z^{*}$ or $z^{*}<y<z$ and else increases.

This means the equilibrium line $y=z^{*}$ is stable (see Fig. 2.).

It should be noticed that the population in the model is infinite. In nature random processes such as genetic 
drift will cause small perturbations from the equilibrium state. It is therefore important to ask whether the population is expected to move along the equilibrium line towards hermaphroditism or dioecy. We have studied this both in simulations and analytically but no evidence has been found for a systematic tendency towards either hermaphroditism or dioecy. The thorough analysis of this problem is rather complex and falls beyond the scope of this paper.

In section $\mathrm{A} 2$ a variable $\theta$ is considered. We could not find analytic expressions for the equilibria in this situation. Numerical studies show that with a variable $\theta$, trioecy is never stable, and that the final population is always either pure male, polymorphic male/hermaphrodite, pure hermaphrodite or polymorphic female/hermaphrodite.

By linearizing equation (11) in some special cases, a qualitative prediction of the equilibrium state of the population can be made. If we denote the value of $\theta$ in generation $i$ by $\theta_{i}$, then it can be shown by equation (11) that, in a population with only males, females can invade the population if

$\prod_{i} \frac{1}{2} \theta_{\mathrm{i}}>1$

and hermaphrodites can invade if

$\prod z+\frac{1}{2} \theta_{i}(1-z)>1$.

In a population with only hermaphrodites, males can invade the population if

$\prod_{i} \frac{z+\frac{1}{2} \theta_{i}(1-z)}{z\left(z+\theta_{i}(1-z)\right)}>1$

and females can invade if

$\prod_{i} \frac{\frac{1}{2} \theta_{i}}{z+\theta_{i}(1-z)}>1$.

If condition (14) is not true, (13) cannot be true either and a pure male (i.e. imperfect) population is expected. If both (15) and (16) are untrue a pure hermaphroditic population (as found abundantly in nature) is expected.

\section{The dynamics of the system of recurrence relations in a homothallic population}

For all types $j$ considered in section B of this study (see Table 1 and Fig. 1.) formula (1) becomes

$$
W x_{1}^{\prime}=x_{1}\left[1+\frac{1}{2} \theta \frac{(1-s)(1-z) x_{2}+x_{3}}{z\left(x_{2}+x_{4}\right)+x_{1}}\right]
$$

$$
\begin{aligned}
W x_{2}^{\prime}= & x_{2}\left\{z\left[1+\frac{1}{2} \theta \frac{(1-s)(1-z) x_{2}+x_{3}}{z\left(x_{2}+x_{4}\right)+x_{1}}\right]\right. \\
& \left.+\frac{1}{2} \theta(1-z)[1-s(1-2 d)]\right\} \\
W x_{3}^{\prime}= & x_{3} \frac{1}{2} \theta \\
W x_{4}^{\prime}= & x_{4}\left\{z\left[1+\frac{1}{2} \theta \frac{(1-s)(1-z) x_{2}+x_{3}}{z\left(x_{2}+x_{4}\right)+x_{1}}\right]+\theta(1-z) d\right\}
\end{aligned}
$$

$W x^{\prime}{ }_{5}=x_{5} \theta d$

where,

$$
\begin{aligned}
W= & (1-\theta)\left[z\left(x_{2}+x_{4}\right)+x_{1}\right] \\
& +\theta\left\{1-(1-d)\left[(1-z)\left(s x_{2}+x_{4}\right)+x_{5}\right]\right\} .
\end{aligned}
$$

It is easy to see that for $d<0.5$ types $2(\mathbf{u i})$ and $3(\mathbf{u})$ will always do better than types $4(\hat{\mathbf{u}} \mathbf{i})$ and $5(\hat{\mathbf{u}})$, and vice versa for $d<0.5$.

With a variable $\theta$, as considered in section $\mathrm{B} 2$, for $d<0.5$ conditions comparable to (13)-(16) in Appendix II can be derived. The same stable population compositions are possible as in a heterothallic species.

Denoting by $\theta_{i}$ the value of $\theta$ in generation $i$, it can be derived from $(17 a),(17 b)$ and $(17 c)$ that in a population with only males:

females can invade the population if

$\prod_{i} \frac{1}{2} \theta_{i}>1$

and hermaphrodites can invade if

$\prod_{i}\left\{z+\frac{1}{2} \theta_{i}(1-z)[1-s(1-2 d)]\right\}>1$.

In a population with only hermaphrodites, males can invade the population if

$\prod_{i} \frac{z+\frac{1}{2} \theta_{i}(1-s)(1-z)}{z\left\{z+\theta_{i}(1-z)[1-(1-d) s]\right\}}>1$

and females can invade if

$\prod_{i} \frac{\frac{1}{2} \theta_{i}}{z+\theta_{i}(1-z)[1-(1-d) s]}>1$.

It is easy to see that in the case of $d>0.5$ there is competition between the types $\mathbf{i}$, $\hat{\mathbf{u}} \mathbf{i}$, and $\hat{\mathbf{u}}$ only. If $x_{2}=x_{3}=0$, formulas $(17 \mathrm{a}),(17 \mathrm{~d})$ and $(17 \mathrm{e})$ can be 
rewritten as

$$
\begin{aligned}
& W x_{1}^{\prime}=x_{1}=a_{1} \cdot x_{1} \\
& W x_{4}^{\prime}=[z+(1-z) \theta d] \cdot x_{4}=a_{4} \cdot x_{4} \\
& W x_{5}^{\prime}=\theta d \cdot x_{5}=a_{1} \cdot x_{1}
\end{aligned}
$$

where all $a_{j}$ are constant if $\theta$ is constant.

If $\theta d>1$ then $a_{5}>a_{4}>a_{1}$ and if $\theta d<1$ then $a_{1}>a_{4}>a_{5}$. Parameter $a_{4}$ can never have the highest value. Therefore either type $\mathbf{i}$ or type $\hat{\mathbf{u}}$ will go to fixation in the situation described in section B1.
If $\boldsymbol{\theta}_{i}$ varies (as described in section B2), then $a_{i, j}$ (the value of $a_{j}$ in generation $i$ ) also varies and after $T$ generations:

$V x_{j}^{(T)}=\prod_{i=0}^{T} a_{i, j} \cdot x_{j}=b_{j} \cdot x_{j}$.

As the $b_{j}$, the product of the $a_{i, j}$ over $T$ generations, are new constants, a system analogous to equation (22) is obtained. This time, however, each of the three types may become fixed, because, with variable $a_{i, j}$ all $b_{j}$ can have the largest value. 